\title{
The Rate of Turbulent Kinetic Energy Dissipation in a Turbulent Boundary Layer on a Flat Plate
}

\begin{abstract}
Sutardi $^{1}$
Abstract-The rate of turbulent kinetic energy dissipation ( $\varepsilon$ is an important parameter in the turbulent flows, such as pipe flows, channel flows, atmospheric turbulence, ocean turbulence, and turbulent boundary layer flows. This study is concerning in the evaluation of the rate of turbulent kinetic energy dissipation in turbulent boundary layers developing on a flat plate. In this study, $\varepsilon$ is obtained simply from the calculation using Taylor's frozen hypothesis. The study is performed experimentally using a low speed wind tunnel with a squared test section of $91 \times 91 \times 540 \mathrm{~cm}$. The maximum attainable freestream velocity is approximately of $15 \mathrm{~m} / \mathrm{s}$ with freestream turbulence intensity is less than $0.5 \%$. Instantaneous fluid velocity is measured using a hot-wire anemometry system connected to a data acqusition and a personal computer. The experiments are performed at freestream velocities of $2.0 \mathrm{~m} / \mathrm{sec}$ and $5.5 \mathrm{~m} / \mathrm{sec}$ corresponding with momentum thickness Reynolds numbers (R $\theta$ ) of approximately 1000 and 3000, respectively. The results show that maximum value of $\varepsilon$ is at approximately $1<y+<10$ at both Reynolds numbers. The results of $\varepsilon$ in the smooth-wall flat plate boundary layer are compared to that in the boundary layer on the flat plate modified with a square groove. There is a slight difference between $\varepsilon$ in the smooth-wall flat plate boundary layer and that in the boundary layer on the flat plate with a square groove.
\end{abstract}

Keywords - turbulent kinetic energy dissipation, Taylor's frozen hypothesis, turbulent boundary layer, hot-wire anemometry, square groove.

\section{INTRODUCTION}

$\mathrm{T}$ urbulent fluid flow is mainly characterized by following fiatures: high Reynolds number, high levels of vorticity fluctuations, high levels of momentum and energy transfers, and dissivative. The dissipative nature of turbulent flows is caused by the action of fluid viscosity. Combination of the fluid viscosity and velocity gradient in the flow results in viscous stresses. The presence of the viscous stress leads to an increase in internal energy due to the deformation work. As a results, there is a reduction in turbulent kinetic energy. Based on this phenomenon, then the energy dissipation due to the action of fluid viscosity is frequently referred to as turbulent kinetic energy dissipation $(\varepsilon)$.

According to Kolmogorov theory, small scales in turbulent flow depend on the fluid kinematic viscosity $(v)$ and the rate of turbulent kinetic energy dissipation. Based on the dimensional argument, one can obtain a small scale turbulence, $\eta$, expressed as

$\eta=(\nu 3 / \varepsilon) 1 / 4$.

The parameter $\eta$ is then called as Kolmogorov length scale. Richardson [1] potulated an energy cascade concepts that in turbulent flows there is energy transfer from large scales (large eddies) through smaller eddies, and to smallest scale eddies. In the case local Reynolds number is very small, than the dissipation of kinetic energy into heat due to the viscosity is very important.

The rate of turbulent kinetic energy dissipation $(\varepsilon)$ can also be used to estimate other Kolmogorov's scales, such as time $(\tau)$ and velocity $(\mathrm{v})$,

$\tau=(v / \varepsilon) 1 / 2$,

and

$v=(v \varepsilon) 1 / 4$.

\footnotetext{
${ }^{1}$ Sutardi is with Department of Mechanical Engineering, Faculty of Industrial Engineering, Institut Teknologi Sepuluh Nopember, Surabaya, 60111, Indonesia. E-mail: sutardi@me.its.ac.id.
}

The rate of turbulent kinetic energy dissipation $(\varepsilon)$ is a function of fluid kinematic viscosity $(v)$ and gradients of fluctuating velocity components. Schlichting [2] showed that $\varepsilon$ can be expressed as

$$
\begin{aligned}
& \varepsilon=v\left[2 \overline{\left(\frac{\partial u}{\partial x}\right)^{2}}+\overline{\left(\frac{\partial v}{\partial y}\right)^{2}+2\left(\frac{\partial w}{\partial z}\right)^{2}}+\overline{\left(\frac{\partial u}{\partial y}+\frac{\partial v}{\partial x}\right)^{2}}\right. \\
& \left.+\overline{\left(\frac{\partial u}{\partial z}+\frac{\partial w}{\partial x}\right)^{2}}+\overline{\left(\frac{\partial v}{\partial z}+\frac{\partial w}{\partial y}\right)^{2}}\right]
\end{aligned}
$$

In eq. (4) it is seen that $\varepsilon$ is a very involved equation; $\varepsilon$ consists of nine fluctuating velocity gradients independently. In most practical situations, however, it is not easy to obtain all nine components using sophisticated instruments such as hot-wire anemometer (HWA), laser Doppler velocimeter (LDV), or particle image velocimeter (PIV). If the turbulence is assumed to be homogeneous and isotropic, eq. (4) can be simplified as [4]

$\varepsilon=15 v \overline{(\partial u / \partial x)^{2}}$

Nowadays, konwledge of the rate of turbulent kinetic energy dissipation $(\varepsilon)$ plays an important role in the study of turbulent flow, especially in the study of turbulence models. In the traditional $\mathrm{k}-\varepsilon$ model, the transport equation of $\varepsilon$ can be expressed as [3]

$\frac{D \varepsilon}{D t}=\frac{1}{\rho} \frac{\partial}{\partial x_{j}}\left(\frac{\mu_{t}}{\sigma_{\varepsilon}} \frac{\partial \varepsilon}{\partial x_{j}}\right)+C_{\varepsilon 1} \frac{\mu_{t}}{\rho} \frac{\varepsilon}{k}\left(\frac{\partial U_{i}}{\partial x_{j}}+\frac{\partial U_{j}}{\partial x_{i}}\right) \frac{\partial U_{i}}{\partial x_{j}}-\frac{C_{\varepsilon 2} \varepsilon^{2}}{k}$

where the absolute turbulent viscosity, $\mu \mathrm{t}$, is expressed as:

$\mu_{t}=\frac{C_{\mu} \rho k^{2}}{\varepsilon}$

Coefficients $\sigma \mathrm{k}, \sigma \varepsilon, \mathrm{C} \varepsilon 1, \mathrm{C} \varepsilon 2$, dan $\mathrm{C} \mu$, are obtained experimentally for particular cases such as boundary layer flow, grid turbulence, jet flows and so on. Together with transport equation of turbulent kinetic energy, $\mathrm{k}$, the transport equation of turbulent kinetic energy dissipation 
rate (Eq. 6) becomes a more famous two-equation turbulent model, i. e. k- $\varepsilon$ turbulent model.

In a very hypothetical case where there turbulence is homogeneous and isotrpic, the rate of of turbulent kinetic energy dissipation can be expressed as in eq. (4). The value of is obtained experimentally using Taylor's frozen hypothesis concepts. Mathematically, Taylor's hypothesis can also be expressed as:

$\frac{\partial u}{\partial x}=-\frac{1}{U(y)} \frac{\partial u}{\partial t}$

where usually obtained from instantaneous velocity measurement, and $\mathrm{U}(\mathrm{y})$ is local mean velocity. The purpose of present study is to evaluate the rate of turbulent kinetic energy dissipation $(\varepsilon)$ in a turbulent boundary layer developing on a flat plate using a hotwire anemometer system. The results are then compared to that occurs in a turbulent boundary layer modified using a single transverse square groove.

\section{METHOD}

The study was performed experimentally using a low speed wind tunnel (Fig. 1). Downstream of the wind tunnel blower is installed a screened diffuser and connected to a 5:1 contraction. Air flow in the test section is driven by a blower and controlled by motorized variable angle inlet vanes. Test section of this tunnel is squared of $91 \times 91 \mathrm{~cm}$ and approximatelly is $540 \mathrm{~cm}$ long. Total length of the tunnel is no less than 20 $\mathrm{m}$. The maximum freestream velocity in the tunnel is approximately of $15 \mathrm{~m} / \mathrm{s}$ and freestream turbulence intensity is less than $0.5 \%$. Instantaneous air velocity is measured using a hot-wire anemometry system connected to a data acquisition and connected to a personal computer. The hot-wire can be transversed normal to the tunnel wall, from the tunnel centerline toward the tunnel wall up to a distance as small as 0.05 $\mathrm{mm}$.

The probe of hot-wire is a tungsten wire with diameter of $5 \mu \mathrm{m}$ platinum plated, single nornal wire, connected to Dantec 55M01 standard bridge. The active wire length is approximately $1 \mathrm{~mm}$ providing the wire length to diameter is more than 200 . The hot wire was calibrated using TSI velocimeter at the tunnel centerline, and a third order polynomial calibration curve is used to fit the data.

The experiments were performed at freestream velocities of $2.0 \mathrm{~m} / \mathrm{sec}$ and $5.5 \mathrm{~m} / \mathrm{sec}$ corresponding with momentum thickness Reynolds numbers (R $\theta)$ of approximately 1000 and 3000, respectively, where $\theta$ is momentum thickness at a location about $250 \mathrm{~cm}$ downstream of the boundary layer tripping wire. At $\mathrm{R} \theta=$ 1000, sampling frequencies were set at $4000 \mathrm{~Hz}$, and the sampling time was $10 \mathrm{sec}$. Therefore, there were 40000 data to construct an instantaneous velocity signal. At R $\theta$ $=3000$, on the other hand, sampling frequencies were set at $6000 \mathrm{~Hz}$, the sampling time was $10 \mathrm{sec}$, and as many as 40000 data were recorded for further analysis.

The measurements were made on a smooth-wall flat plate and with a transverse square groove located $2.5 \mathrm{~m}$ from the leading edge (Fig. 1). The boundary layer was tripped at the leading edge of the plate using a roughness element made of $100 \mathrm{~mm}$ wide sandpaper (series 0811) and a $1.5 \mathrm{~mm}$ diameter cylindrical rod. The flat plate is made of $25 \mathrm{~mm}$ thick acrylic and is mounted horizontally on the floor of the wind tunnel. The groove depth $(d)$ is 5 $\mathrm{mm}$, and depth to width ratio $(d / w)$ is unity.

\section{RESULT AND DISCUSSION}

\section{A. Instantaneous Velocity Signal and Its Derivatives}

Figure 2 shows a typical instantaneous velocity fluctuation in a turbulent boundary layer developing on a smooth-wall flat plate. It was obtained from a boundary layer with a freestream velocity of $2.0 \mathrm{~m} / \mathrm{s}$, corresponds with flow Reynolds number ( $\mathrm{R} \theta$ ) based on momentum thickness of approximately 830 . This thickness of the boundary layer at that location is approximately $95 \mathrm{~mm}$, and the signal was taken at $y / \delta=0.0274$. If the signal shown in Fig. 2 is converted into the rate of change of instantaneous velocity fluctuation $(\partial \mathrm{u} / \partial \mathrm{t})$, one obtained a signal as shown in Fig. 3.

Furthermore, converting the time derivative signal (Fig. 3) using Taylor's frozen hypothesis results in a spatial derivative of instantaneous velocity fluctuation as shown in Fig. 4. For the instantaneous velocity fluctuation signal shown in Fig. 2, the corresponding values of local mean velocity, $\mathrm{U}$, , and $\varepsilon$ are summarized in Table 1.

\section{B. Turbulent Kinetic Energy Dissipation in the Boundary Layer}

Figures 5 and 6 show the distribution of across the boundary layer at $\mathrm{R} \theta=1000$ and 3000 , respectively. In those figures, the distribution of in the smooth-wall flat plate boundary layer is compared to that in the boundary layer disturbed by a single transverse square groove.

At lower $\mathrm{R} \theta$, there is a significant difference in on for the two wall configutaions. The maximum value of for the smooth-wall is about 0.17 /s and occurs at $\mathrm{y}+$ about 2.0, while the maximum value of for the wall modified by the transverse square groove is about 0.12 and occurs at $\mathrm{y}+$ about 4.0. At higer $\mathrm{R} \square,(\mathrm{R} \theta=3000)$, similarity in for the case of smooth-wall and the modified wall boundary layer is very good. All data collapse very well throughout the layer. At the higher $\mathrm{R} \square$, the maximum value of is approximately $0.18 / \mathrm{s}$ and occurs at $\mathrm{y}+\approx 4.0$.

Good agreement in for the two different wall geometries at higher $\mathrm{R} \theta$ is not followed by that one in the lower $\mathrm{R} \theta$. At lower $\mathrm{R} \theta$, there is a big difference in for the smooth-wall compared to that for wall modified by a transverse square groove in the region $y+\leq 200$. The reasonable explanation for this discrepancy is not clear at this moment.

\section{Discussion about the Rate of Turbulent Kinetic} Dissipation

In most practical application, it is rarely found homogeneous and isotropic turbulence. Hence, the use of homogeneous and isotropic turbulence concept to calculate the rate of turbulent kinetic dissipation $(\varepsilon)$ is an hypothetical approach. It is used to simplify the problem, since it is difficult to obtain all derivatives as stated in eq. (4) to obtain.

Perot and Natu [5] showed that the distribution of $\varepsilon 11$, i.e. the rate of turbulent kinetic dissipation for streamwise velocity fluctuation, for a channel flow is somewhat similar qualitatively with present results. They were doing the work using numerical study. Peak 
value of $\varepsilon 11$ was also at location close to the channel wall.

Charuchittipan and Wilson [6] tried to obtain $\varepsilon$ from composite spectrum. They also used the Taylor frozen hypothesis concept for transformation Kolmogorov's wave spectrum and they show that $\varepsilon$ is a function of spectral density frequency (f), local mean velocity (U), power spectra $(\mathrm{Su}(\mathrm{f}))$, and two coeffcients, say, T11 and au as:

$\varepsilon=\frac{2 \pi}{U}\left[\frac{f^{5 / 3} S_{u}(f)}{T_{11} \alpha_{u}}\right]^{3 / 2}$

In eq. (9), T11 is a factor defined as [6]

$T_{11}=1-\frac{\overline{u^{2}}}{9 U^{2}}+\frac{2 \overline{v^{2}}}{3 U^{2}}+\frac{2 \overline{w^{2}}}{3 U^{2}}$

where $\mathrm{u}, \mathrm{v}$, and $\mathrm{w}$ are velocity fluctuating components in the $\mathrm{x}, \mathrm{y}$, and $\mathrm{z}$ directions, respectively. Meanwhile, Charuchittipan and Wilson [6] proposed $\alpha \mathrm{u}=0.5$.

Poggi and Katul [7] calculated $\varepsilon$ from several methods. They showed that the results of $\varepsilon$ based on they calculation as a function of the distance from the wall (floor). The results of present study (Figs. 5 and 6) are qualitatively similar to that obtained by Poggi and Katul [7].

\section{CONCLUSION}

Study of turbulent kinetic energy dissipation rate has been studied in a turbulent boundary layer, both for smooth-wall flat plate and for flat plate modified using a single transverse square groove. The study used a hot wire anemometer system to obtain instantaneous velocity fluctuation and is performed in a low speed wind tunnel at two Reynolds numbers, $R \theta=1000$ and 3000. Some conclusions can be summarized as follow.

1. Turbulent kinetic energy dissipation rate, $\varepsilon$, is calculated from assumption of homogeneous isotropic turbulence.

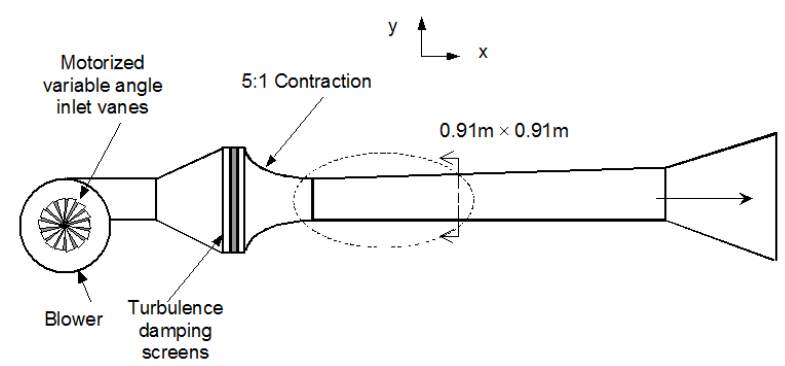

a). Wind tunnel with its components

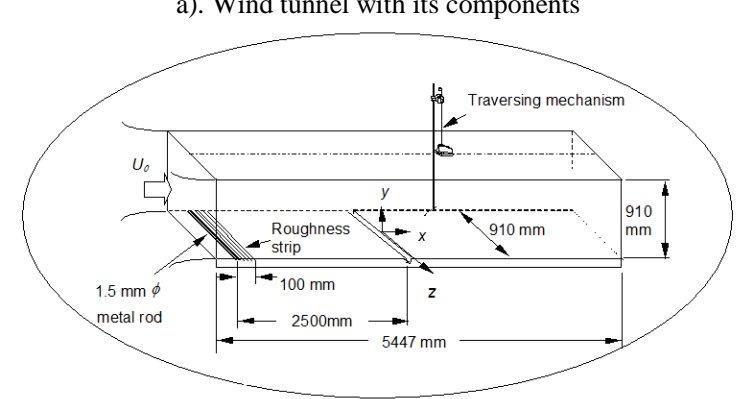

b). Wind tunnel test section details
2. There is a slight difference between $\varepsilon$ in the smoothwall flat plate boundary layer and that in the boundary layer developing on the flat plate modified with a square groove for $\mathrm{R} \theta=3000$ in the range $\mathrm{y}+$ $<3$.

3. The difference in $\varepsilon$ becomes more significant for $\mathrm{R} \theta$ $=1000$, and almost occupies the whole layer of the boundary layer thickness.

4. The location of maximum value of $\varepsilon$ is at approximately $1<\mathrm{y}+<10$ at both Reynolds numbers and for either smooth-wall flat plate or for flat plate modified using a single transverse square groove.

\section{ACKNOWLEDGEMENT}

I thank do Faculty of Engineering, Memorial University of Newfoundland, Canada, for providing wind tunnel test facility for doing experimental study and also I thank Prof. C.Y. Ching for giving enthusiastic discussion about the topic.

\section{REFERENCES}

[1] L. F. Richardson, Weather Prediction by Numerical Process, Cambridge, Cambridge Univ. Press, 1922.

[2] [2] H. Schlichting, Boundary Layer Theory, seventh ed., New York, McGraw-Hill Book Company, 1979.

[3] [3] H. H. Bruun, Hot-Wire Anemometry: Principles and Signal Analysis, first ed., Oxford, Oxford University Press, 1995.

[4] [4] J. O. Hinze, Turbulence, second ed., New York, McGrawHill, 1975.

[5] [5] B. Perot and S. Natu, "A model for the dissipation rate tensor in inhomogeneous and anisotropic turbulence", Phys. Fluids, vol. 16(11), pp. 4053-4065, 2004.

[6] [6] D. Charuchittipan and J. D. Wilson, "Turbulent kinetic energy dissipation in the surface layer", Boundary-Layer Meteorol., vol. 132, pp. 193-204, 2009.

[7] [7] D. Poggi and G. G. Katul, "Evaluation of the turbulent kinetic energy dissipation rate inside canopies by zero- and level-crossing density method", Boundary-Layer Meteorol., vol. 136, pp. 219-233, 2010.
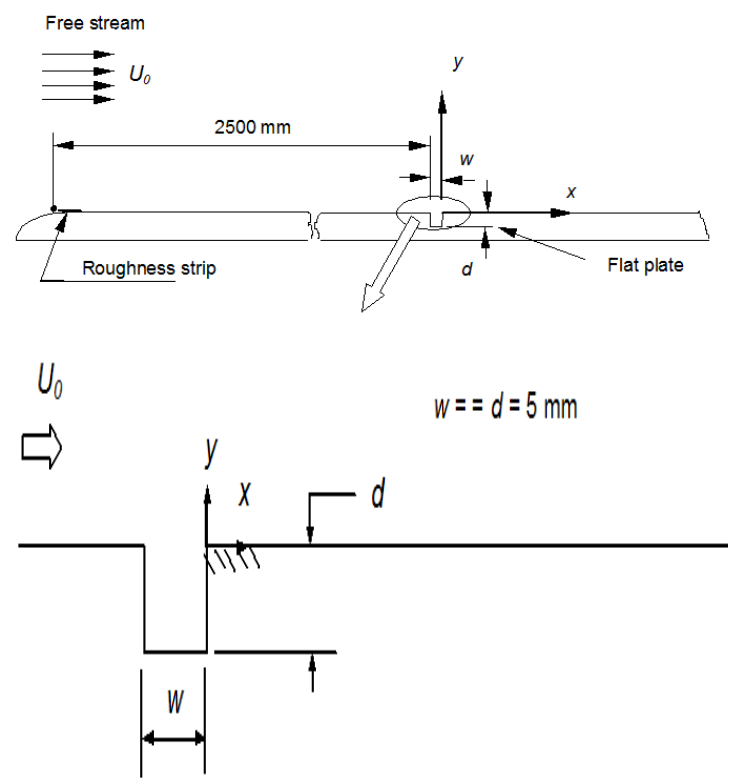

c). A schematic of test-plate showing a single transverse groove 


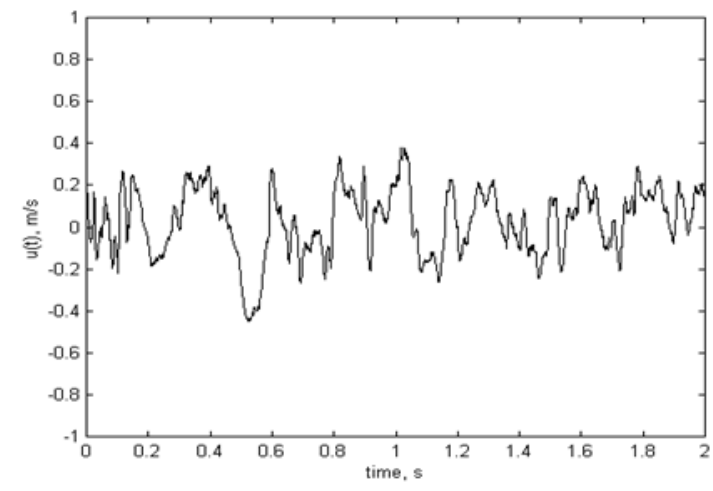

Figure 2. Instantaneous streamwise velocity fluctuation. $U_{0}=1.40 \mathrm{~m} / \mathrm{s}$, $\mathrm{y} / \delta=0.0274, \delta=95 \mathrm{~mm}$

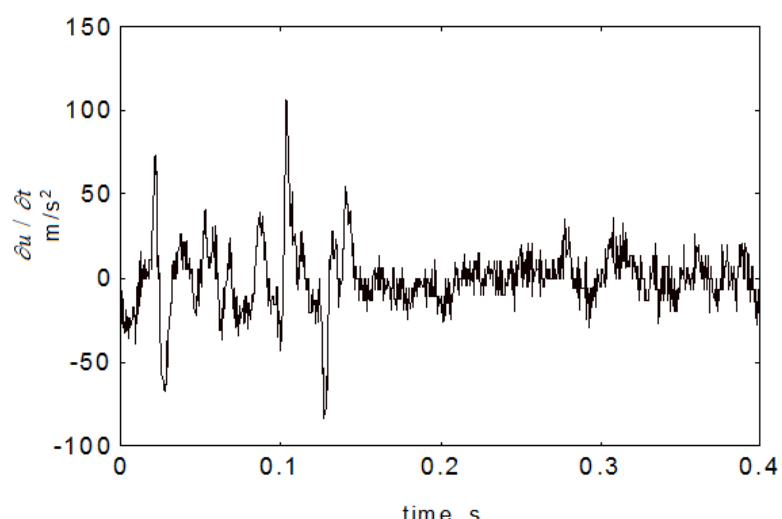

Figure 3. Bacterial viability in copper containing cultures of (a) B. cereus ATCC 1178 (b) B. cereus ATCC 9632

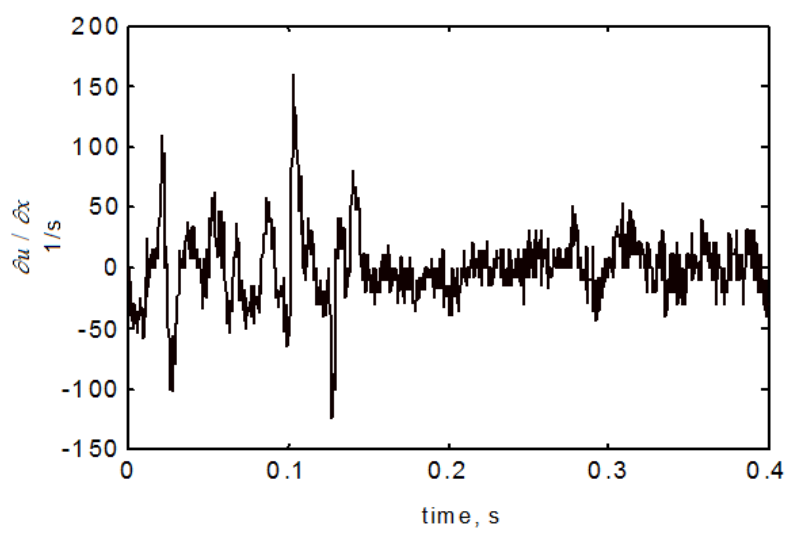

Figure 4. Spatial derivative of streamwise velocity fluctuation shown in Fig. 2

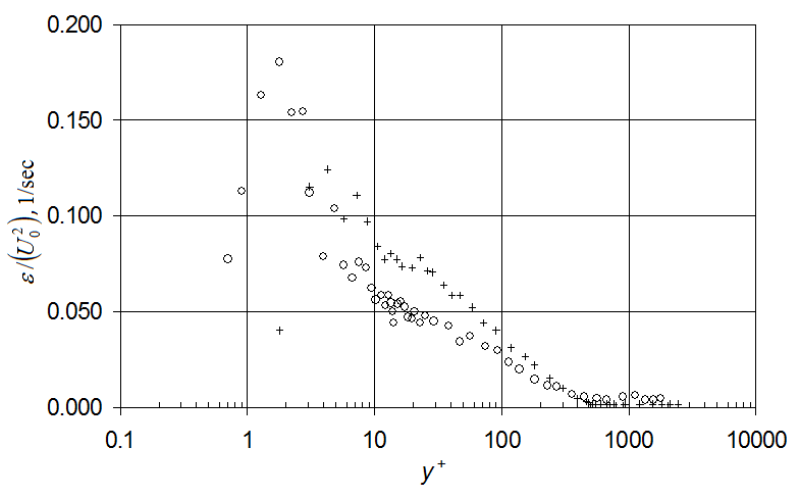

Figure 5. Rate of the turbulent kinetic energy distribution at $R_{\theta}=1000$ Symbols: $\circ$, smooth-wall; + , wall with a transverse square groove

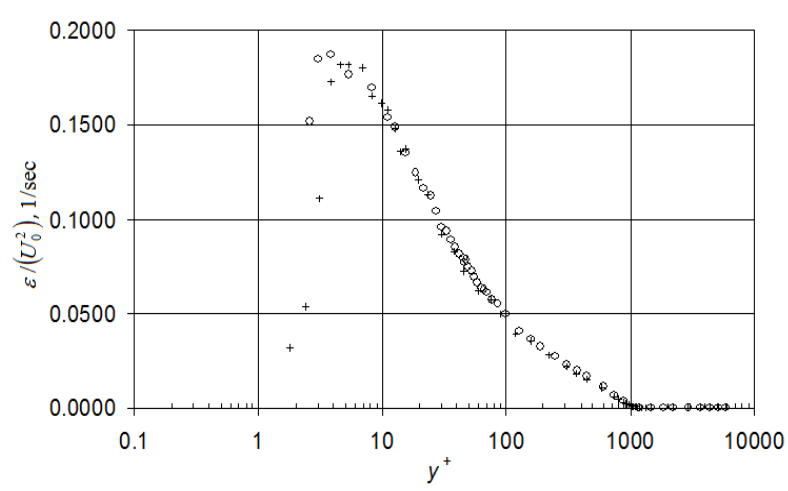

Figure 6. Rate of the turbulent kinetic energy distribution at $R_{\theta}=3000$ Symbols: $\circ$, smooth-wall; + , wall with a transverse square groove

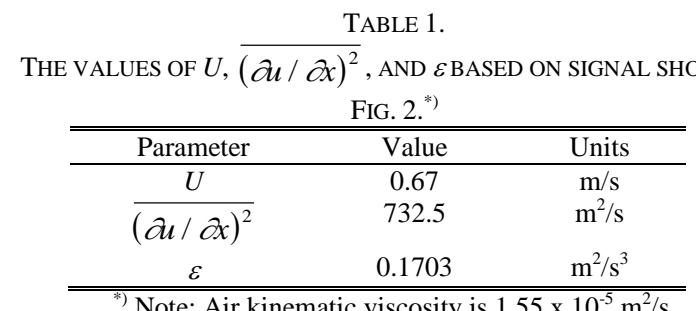

${ }^{*}$ Note: Air kinematic viscosity is $1.55 \times 10^{-5} \mathrm{~m}^{2} / \mathrm{s}$. 